\title{
Apuntes sobre la protección de los conocimientos tradicionales'
}

\author{
Por Anáili Suárez Castro, Yarely Centeno Miranda, Daniel Noa Sánchez y Eugenio Izquierdo Rodríguez
}

Resumen: El conocimiento tradicional está integrado por diferentes elementos que comúnmente se designan como conocimientos indígenas o tribales, folklore, medicina tradicional, etc. En estos momentos resulta una necesidad imprescindible saber en profundidad las regulaciones que existen en relación con este tema de la propiedad intelectual y los recursos genéticos, conocimientos tradicionales y folklore. En este trabajo se exponen aspectos básicos existentes en el debate internacional que tienen lugar bajo el auspicio de la Organización Mundial de la Propiedad Intelectual (OMPI).

Palabras clave: Conocimiento tradicional, Propiedad intelectual, Mecanismos de protección, Folklore.

\section{Title: Notes about the protection of the traditional knowledge}

Abstract: The traditional knowledge is integrated by different elements designed as "indigenous or tribal knowledge, folklore, traditional medicine knowledge, and others". Nowadays it is an essential need to know the existing regulations related to the intellectual property, genetic resources, traditional knowledge and folklore. In this work there are basic aspects related with the international discussion that takes place under the sponsorship of the World Intellectual Property Organization (WIPO).

Keywords: Traditional knowledge, Intellectual property, Protection mechanisms, Folklore.

\section{Introducción}

EL CONOCIMIENTO TRADICIONAL está integrado por diferentes elementos que comúnmente se designan como "conocimientos indígenas o tribales, folklore, conocimientos de medicina tradicional, etc." Sin embargo no debe verse necesariamente como algo ancestral o de épocas remotas; en él está involucrado en todo momento un proceso periódico y casi diario de creación de los individuos y las comunidades en el enfrentamiento a los retos que les plantea su entorno físico y social.

De muchas maneras el conocimiento tradicional es también contemporáneo y las ventajas comerciales o de otro tipo que se deriven de su uso deben propiciar la preservación de los derechos de propiedad intelectual que se implican en el comercio internacional, las comunicaciones y los intercambios culturales.

En la literatura consultada existen varias definiciones de este término y la que nos brinda Teodora Zamudio nos parece la más adecuada y concreta: "conocimiento tradicional es el saber culturalmen- te compartido y común a todos los miembros que pertenecen a una misma sociedad, grupo o pueblo, y que permite la aplicación de los recursos del entorno natural de modo directo, compuesto, combinado, derivado o refinado, para la satisfacción de necesidades humanas, animales, vegetales y/o ambientales, tanto de orden material como espiritual"3.

Recientemente los conocimientos tradicionales, así como la manera de conservarlos, protegerlos y hacer un uso equitativo de ellos, han sido objeto de atención cada vez mayor en varios debates sobre política en cuestiones tan diversas como agricultura y alimentación, medio ambiente (especialmente conservación de la diversidad biológica), salud (incluidas las medicinas tradicionales), derechos humanos indígenas, política cultural, comercio y desarrollo económico.

\section{La OMPI en la protección de los conocimientos tradicionales}

La Secretaría de la Organización Mundial de la Propiedad Intelectual (OMPI) utilizó el siguiente concepto de "conocimientos tradi- cionales" a fin de efectuar un análisis realista y bien documentado de los aspectos de propiedad intelectual relacionados con la protección de los conocimientos tradicionales y el folklore en misiones exploratorias que llevó a cabo en los años 1998 y 1999 :

"Los conocimientos tradicionales hacen referencia a las obras literarias, artísticas o científicas basadas en la tradición; así como las interpretaciones o ejecuciones; invenciones, descubrimientos científicos; dibujos o modelos; marcas, nombres y símbolos; información no divulgada; y todas las demás innovaciones y creaciones basadas en la tradición que procedan de la propiedad intelectual en el ámbito industrial, científico, literario o artístico. La expresión 'basadas en la tradición' se refiere a los sistemas de conocimiento, creaciones, innovaciones y expresiones culturales que se han transmitido de generación en generación; se considera generalmente que pertenecen a un pueblo en particular o a su territorio y evolucionan constantemente en respuesta a los cambios que se producen en su entorno. Entre las categorías de conocimientos tradiciona- 
les figuran: los agrícolas, científicos, técnicos, ecológicos, medicinales -incluidas las medicinas y los remedios conexos-; sobre diversidad biológica; expresiones del folklore en forma de música, baile, canción, artesanía, dibujos y modelos, cuentos, obras de arte; elementos de los idiomas, como los nombres, indicaciones geográficas y símbolos; y bienes culturales muebles. Quedaría excluido de esta descripción lo que no se derive de la actividad intelectual en el ámbito industrial, científico, literario o artístico, como los restos humanos, los idiomas en general y otros elementos similares del 'patrimonio' en un sentido amplio" 6 .

En los trabajos del bienio 1998-1999 se determinaron las cuestiones objeto de estudio y la evaluación de las necesidades y expectativas en materia de propiedad intelectual de los distintos grupos interesados en la esfera de los conocimientos tradicionales.

Entre 2000 y 2001 la labor exploratoria se centró en actividades prácticas previstas para examinar los enfoques actuales y futuros. Desde el año 2000 las actividades de la $O M P I$ en esta materia trascienden el programa exploratorio y se extienden a los programas de cooperación para el desarrollo, formación y asistencia técnica que se llevan a cabo en el contexto de numerosas partidas principales de su Programa y presupuesto para el bienio 2000-2001.

En el $26^{\circ}$ período de sesiones de la Asamblea General de la OMPI, celebrado del 25 de septiembre al 3 de octubre de 2000, los estados miembros decidieron crear un órgano especial para examinar cuestiones de propiedad intelectual sobre recursos genéticos, conocimientos tradicionales y folklore. Este órgano, el Comité Intergubernamental sobre Propiedad Intelectual y Recursos Genéticos, Conocimientos Tradicionales y Folklore, celebró su primera sesión en Ginebra del 30 de abril al 3 de mayo de 2001.

Su labor se centra en tres temas principales:

- acceso a los recursos genéticos y distribución de beneficios;

-protección de los conocimientos tradicionales, las innovaciones y la creatividad, estén o no asociados a dichos recursos; y

—protección de las expresiones del folklore, incluidas las artesanías.

La tercera reunión, celebrada en junio de 2002 en Ginebra, fue una expresión de los múltiples criterios y posiciones que mantienen los diferentes estados sobre el tema. En general hay dos posiciones: una defiende que los mecanismos existentes de la propiedad intelectual pueden o ya se han aplicado a los conocimientos tradicionales; la otra insiste en que se debe adoptar un mecanismo nuevo de protección sui generis para el caso particular de los conocimientos tradicionales. Sobre este punto el Comité Intergubernamental debe profundizar en la efectividad de una solución u otra.

\section{Convenio sobre diversidad biológica (CDB)}

El $C D B$ (1993) es el intento más responsable y respetado en el empeño de que se pongan a disposición de la humanidad los recursos genéticos de la biodiversidad de todos los países sobre bases comunes y con las garantías necesarias. Es el primer acuerdo mundial integral que aborda todos los aspectos de la diversidad biológica. Reconoce los derechos soberanos de los estados sobre sus recursos naturales, otorgándoles la facultad de regular el acceso a dichos recursos genéticos, pues hasta ese momento el acceso a ellos se consideraba libre, al formar parte del patrimonio de la humanidad.

Los objetivos del $C D B^{1}$ propugnados en su artículo 1 son:
-Conservación de la diversidad biológica.

—Utilización sostenible de sus componentes.

-Participación justa y equitativa en los beneficios que se deriven de la utilización de los recursos genéticos.

\section{«La 3a reunión del Comité \\ Intergubernamental sobre Propiedad Intelectual y Recursos Genéticos, Conocimientos Tradicionales $y$ Folklore, de la OMPI, (2002) fue una expresión de los múltiples criterios y posiciones que mantienen los diferentes estados sobre el tema»}

En su artículo 8j, estipulado para la conservación in situ, establece que cada parte contratante, en la medida de lo posible y según proceda con arreglo a su legislación nacional, respetará, preservará y mantendrá los conocimientos, las innovaciones y las prácticas de las comunidades indígenas y locales que entrañen estilos tradicionales de vida pertinentes para la conservación y la utilización sostenible de la diversidad biológica y promoverá su aplicación más amplia, con la aprobación y la participación de quienes posean esos conocimientos, innovaciones y prácticas, y fomentará que los beneficios derivados de la utilización de esos conocimientos, innovaciones y prácticas se compartan equitativamente ${ }^{2}$.

El articulo 15b reconoce los derechos soberanos de los estados sobre sus recursos naturales, la facultad de regular el acceso a los recursos genéticos como política de los gobiernos nacionales y está someti- 
da a la legislación nacional con el ánimo de crear condiciones para facilitar a otras partes contratantes el acceso a los recursos genéticos para utilizaciones ambientalmente adecuadas, y no imponer restricciones contrarias a los objetivos del convenio.

El acceso a los recursos genéticos estará sometido al consentimiento fundamentado previo de las partes contratantes del $C D B$ que proporcionan los recursos, a menos que esa parte decida otra cosa, y tomara medidas legislativas, administrativas o de política, según proceda, para compartir en forma justa y equitativa los resultados de las actividades de investigación y desarrollo así como los beneficios derivados de la utilización comercial y de otra índole de los recursos genéticos con la parte contratante que los aporta. Esta participación se llevará a cabo en condiciones mutuamente acordadas.

En el párrafo 5 del artículo 16 se declara asimismo que las partes contratantes, reconociendo que las patentes y otros derechos de propiedad intelectual pueden influir en la aplicación del convenio, cooperarán a este respecto de conformidad con la legislación nacional y el derecho internacional para velar para que esos derechos apoyen y no se opongan a los objetivos del Convenio.

En cuanto a Cuba, Citma (Ministerio de Ciencia, Tecnología y Medio Ambiente) y la Oficina $\mathrm{Cu}$ bana de la Propiedad Industrial $(O C P I)$ respectivamente, trabajan con sus expertos en la atención de los aspectos normativos para la aplicación en el país de los acuerdos del $C D B$. Desde el punto de vista de este convenio, está vigente en el país la Resolución 111 que en su capítulo II, artículos 9 al 14, regula cómo debe realizarse el acceso a la diversidad biológica nacional. En estos momentos se está elaborando el proyecto de Ley del con- sentimiento previo fundamentado, que debe dar respuesta total a lo planteado en el artículo $15 \mathrm{~b}$ de la $C D B^{7}$.

\section{Los Adpic de la OMC}

Los Adpic (Aspectos de propiedad intelectual en el comercio) y el $C D B$, deberían respaldarse mutuamente y promover el uso sostenible de los recursos. Habría que introducir algunas modificaciones en el acuerdo sobre los Adpic a fin de que no contradiga los objetivos del $C D B^{4}$.

\section{«En Cuba, Citma y OCPI trabajan en la atención de los aspectos normativos para la aplicación en el país de los acuerdos del CDB»}

El acuerdo de la $O M C-W T O$ (Organización Mundial del Comercio) sobre los Adpic tiene elementos que difieren del $C D B$ sobre los conocimientos tradicionales. A saber: 1.- la concesión de patentes a todas las tecnologías sin tener en cuenta mecanismos de distribución que garanticen los beneficios de países o comunidades poseedoras del conocimiento tradicional o de un material biológico 2.- no tiene disposición alguna respecto el consentimiento previo de los gobiernos para el acceso a recursos biológicos, lo cual seguiría proporcionando el aumento de los monopolios sin tener en cuenta transferencias de tecnología, ni el reconocimiento de los proveedores de recursos y conocimientos de comunidades o países enteros.

En nombre de China, Cuba, Ecuador, India, Pakistán, República Dominicana, Tailandia, Venezuela, Zambia, Zimbabwe y propio, Brasil presentó el 21 de junio de 2002 una propuesta para que el consejo de los Adpic recomiende al Comité de Negociaciones Comerciales que adop- te la decisión de modificar el acuerdo sobre los Adpic de manera que estipule que los miembros exijan al solicitante de una patente:

- la divulgación de la fuente y el país de origen del recurso biológico y de los conocimientos tradicionales utilizados en la invención;

- pruebas del consentimiento fundamentado previo mediante la aprobación de las autoridades en el marco de los regímenes nacionales pertinentes; $y$

—pruebas de la distribución justa y equitativa de los beneficios conforme al régimen nacional del país de origen.

De esta forma se podría resolver un problema esencial de coherencia entre los dos acuerdos internacionales vinculantes. Sin duda, las empresas y personas de buena fe cumplidoras de la ley no tendrían dificultades para satisfacer los requisitos mencionados; muchas participan ya en contratos bilaterales que incluyen tales prescripciones. En consecuencia, la modificación que se propone tendría la ventaja clara de proporcionar unas condiciones previsibles a los gobiernos, los inversores, las comunidades tradicionales y los investigadores. De esta manera se alentaría la investigación y el desarrollo biotecnológicos en los países en desarrollo, lo cual estaría en consonancia con los objetivos del acuerdo sobre los $A d$ pic de promover la innovación tecnológica y la transferencia y difusión de tecnología?.

\section{Conocimientos tradicionales vinculados a sistemas de propiedad intelectual}

El conocimiento tradicional que ha producido otros bienes también puede ser protegido y explotado por las comunidades o pueblos indígenas a través de los sistemas de propiedad intelectual. Los modelos de conocimiento tradicional existen en 


\section{baratz}

\section{En primera línea en}

Sistemas de Información y Gestión del Conocimiento
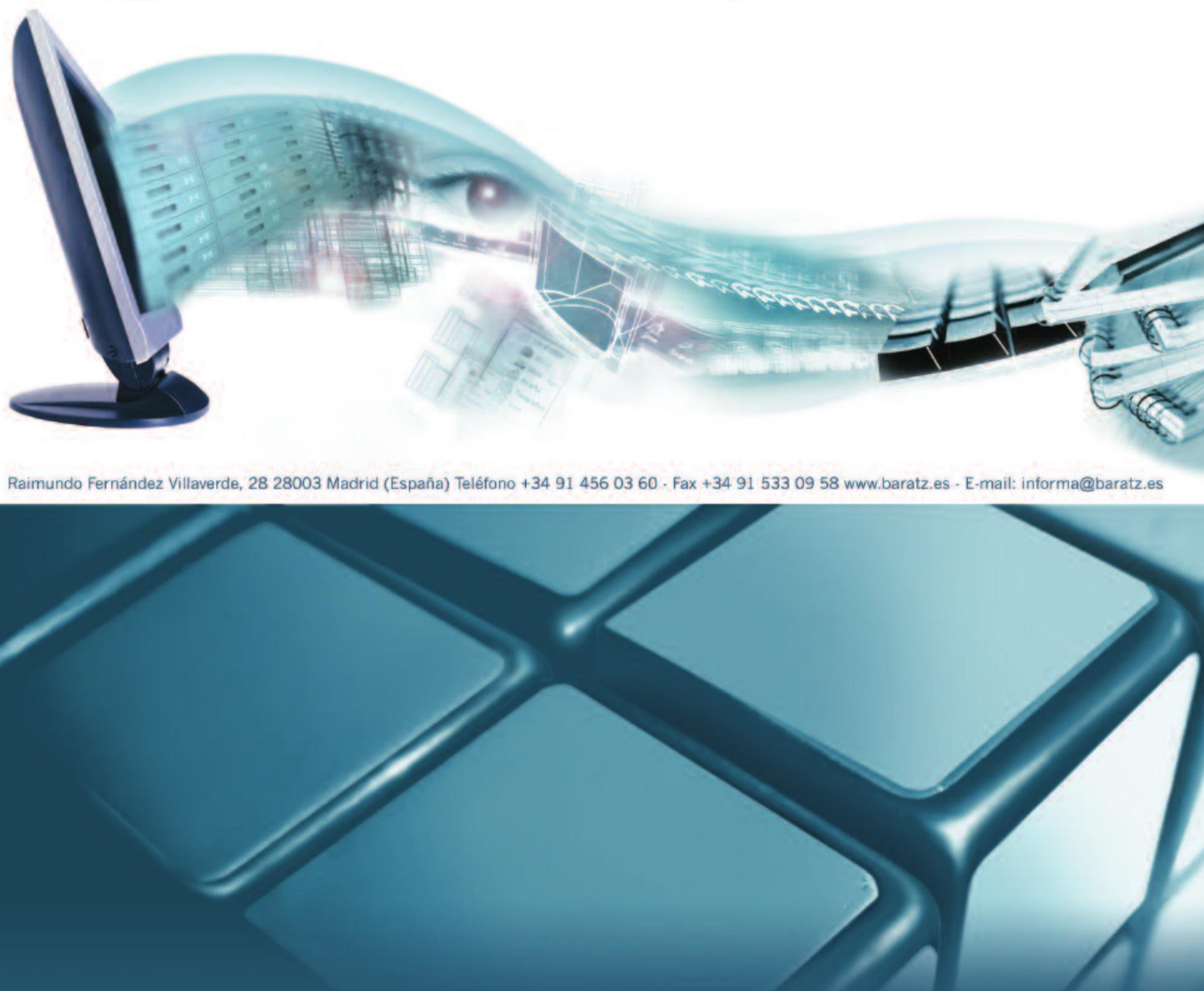

(9) Solvciones para bibliotecas:

Absys, absys NET, Absys express

(9) Gestión documental y del conocimiento: BKM, Baratz Windows, BRSCGI

(2) Soluciones para archivos: Albalá

(2) Servicios de Catalogación Retrospectiva

(9) Edición en CD-Rom

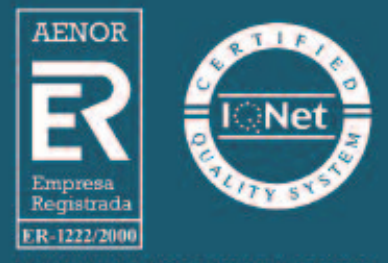


ámbitos como medicina, alimentación, agricultura, gestión medioambiental, conservación de la diversidad biológica, y los objetos culturales, por nombrar sólo algunos.

Otra característica de los conocimientos tradicionales es el sentido de identidad que confieren a la comunidad; son para sus titulares un medio de identificación cultural, de manera tal que su conservación e integridad se vinculan con la preservación de las distintas culturas per se. Una definición de conocimientos tradicionales podría incluir asimismo un elemento que respondiera al derecho reivindicado por los grupos indígenas, las comunidades locales y cualquier otro titular de ellos, de determinar por sí mismos lo que constituyen sus propios conocimientos, innovaciones, y prácticas, así como el modo en que deben definirse.

\section{«La investigación en el campo de los productos naturales en la actualidad no puede desarrollarse sin un conocimiento profundo de estos temas»}

Algunos sistemas identificados con esta temática son de varios países que aplican los mecanismos de propiedad intelectual existentes para la protección de conocimientos tradicionales (la Unión Europea, Suiza, Turquía, Canadá, Australia y Rusia) ${ }^{5}$. Su aplicación, generalmente, se ha basado en las leyes de derecho de autor y en la protección de dibujos o modelos industriales.

En otros países (Guatemala, Perú, Panamá) se han adoptado leyes especiales con el fin de proteger los conocimientos tradicionales en virtud de un nuevo sistema. En estos territorios el gobierno es quien concede los derechos y los registra de manera pertinente, quedando esti- pulado además el beneficio de la comunidad.

En Cuba la $O C P I$ viene participando en debates de la OMPI para tomar elementos y definir la estrategia más conveniente para el país; igualmente trabaja coordinadamente con Citma para que la Ley del consentimiento previo fundamentado recoja los elementos básicos que se plantean en este tema ${ }^{7}$.

\section{Consideraciones finales}

Coincidimos con Zamudio en que "no habrá recursos biológicos disponibles a menos que éstos se conserven adecuadamente; por otro lado, habría pocos incentivos para la conservación y la utilización sostenible si los recursos biológicos no generasen beneficios (lo cuales no son sólo los traducibles en valores crematísticos) para las partes interesadas locales y del país de origen, que son los propietarios (o 'disponentes') de la diversidad biológica, además de sus principales custodios"s.

Todavía la $O M P I$ trabaja en la armonización de las diversas opiniones que se manejan en su Comité Intergubernamental sobre Propiedad Intelectual y Recursos genéticos, Conocimientos Tradicionales y el Folklore.

No se ha valorado aún si la protección de los conocimientos tradicionales se hará a través de los mecanismos establecidos por la propiedad intelectual (derecho de autor o propiedad industrial) o la utilización de un sistema sui generis. Ha habido varias reuniones de este comité para llegar a una conclusión que aún está en debate.

En nuestra opinión, ambas son positivas e indispensables y sólo la combinación de los dos sistemas puede llevar a buen término la protección de los conocimientos tradicionales y, por ende, la contribución al desarrollo de la administra- ción y aplicación de los mismos en beneficio de sus creadores.

En los momentos que terminamos este trabajo hemos conocido la celebración en junio de 2005 de la octava sesión del Comité. Esperamos que los resultados contribuyan a dirimir esta cuestión esencial.

\section{Nota}

1. Trabajo presentado como evaluación del Módulo de Invenciones de la Maestría en Propiedad Intelectual de la Oficina Cubana de Propiedad Industrial (OCPI), marzo 2005.

\section{Bibliografía}

1. Acuerdos internacionales. Convenio sobre diversidad biológica. Consultado en: 08-03-05.

http://www.medioambiente.gov.ar/acuerdos/conv enciones/Cdb/dbconven.htm\#ARTÍCULO\%201

2. $C D B$ y conocimiento tradicional. Convenio sobre la diversidad biológica. Consultado en: 08-03-05.

http://www.prodiversitas.bioetica.org/tkcbd.htm

3. Conocimiento tradicional (y propiedad intelectual/industrial). Consultado en: 08-03-05. http://www.indigenas.bioetica.org/tk.htm

4. OMC y conocimiento tradicional. Organización Mundial del Comercio. Consultado en: 08-03-05.

http://www.prodiversitas.bioetica.org/tkomc.htm

5. OMPI. Comité Intergubernamental sobre Propiedad Intelectual y Recursos genéticos, Conocimientos Tradicionales y el Folklore, 2001: Análisis y conclusiones preliminares del estudio sobre formas actuales de protección de los conocimientos tradicionales mediante la propiedad intelectual. Documento OMPI/Grtkf/IC/2/9. Segunda sesión, Ginebra, 10 al 14 de diciembre de 2001, 12 pp.

6. OMPI. Comité Intergubernamental sobre Propiedad Intelectual y Recursos genéticos, Conocimientos Tradicionales y el Folklore. Los conocimientos tradicionales: definiciones y términos. Documento WIPO/Grtkf/IC/3/9. Tercera sesión, Ginebra, 13 a 21 de junio de 2002, 48 pp.

7. Ramírez Mirabal, Rafael. "El Convenio de la diversidad biológica, la protección de los conocimientos tradicionales y el desarrollo de nuevos productos de origen natural". En: IV Congreso Nacional de Farmacología, 2002.

8. Zamudio, Teodora. El Convenio sobre la diversidad biológica en América Latina. Etnobioprospección y propiedad industrial. Notas desde una cosmovisión económico-jurídica, 2005.

Consultado en: 09-03-05.

http://www.prodiversitas.bioetica.org

9. Zamudio, Teodora. Relación entre el acuerdo sobre los Adpic y el Convenio sobre la diversidad biológica y la protección de los conocimientos tradicionales, 2005. Consultado en: 08-03-05.

http://www.prodiversitas.bioetica.org 
Anáili Suárez Castro, Sección de Propiedad Intelectual y Estudios de Mercado, del grupo Gestión de Información del Centro Nacional de Sanidad Agropecuaria (Censa), La Habana, Cuba.

anaili@censa.edu.cu
Yarely Centeno Miranda, Grupo de Ciencia y Técnica del Instituto de Ciencia Animal (ICA), Universidad Agraria de la Habana, La Habana, Cuba.

ycenteno@ica.co.cu

Daniel Noa Sánchez, Universidad de la Habana, La Habana, Cuba. informin@mail.mn.co.cu

Eugenio Izquierdo Rodríguez, Universidad de la Habana, Universidad de Ciencias de la Informática, La Habana, Cuba.

eugenio@uci.cu 